Pacific Journal of Mathematics

ON THE STRUCTURE TOPOLOGY OF SIMPLEX SPACES 


\title{
ON THE STRUCTURE TOPOLOGY OF SIMPLEX SPACES
}

\author{
Alan Gleit
}

\begin{abstract}
This paper studies the hull-kernel topology of the maximal ideal space of separable simplex spaces. We show that the properties of local compactness, first countability, and second countability are equivalent.
\end{abstract}

A simplex space is an ordered Banach space $V$ with closed positive cone whose dual is an $L$-space $[4,5,6]$. Let $P_{1}(V)$ be the positive linear functionals on $V$ with norm less than or equal to one. The pure states are the extreme points of $P_{1}(V)$ and are denoted by $E P_{1}(V)$. We shall write $E^{+}$for the nonzero extreme points of $P_{1}(V)$, i.e.,

$$
E^{-}=E P_{1}(V)-\{0\}
$$

We let $Z$ be the closure, in the weak* topology, of $E^{+}$.

We let $\max V$ be the set of closed maximal ideals of $V$ equipped with the hull-kernel topology. The closed ideals of $V$ are in a one-toone order inverting correspondence with the closed faces of $P_{1}(V)$ containing zero. Thus, $\max V$ may be identified with $E^{+}$as point sets. By this identification we can transfer the hull-kernel topology to $E^{+}$and define a new topology, called the structure topology, on $E^{+}$. Its closed sets are the nonzero extreme points of a closed face containing zero. Hence, the structure topology is weaker than the weak* topology.

In this paper we shall consider various topological properties of $\max V$. In particular, we consider compactness, local compactness, first countability, second countability, and standard Borel structure. It was conjectured in [5] that for separable simplex spaces the latter four properties are equivalent. This is very nearly correct, as we see in Theorem 3.3 and Proposition 3.6.

In section 1, we study the structure topology and introduce several new maps. We give several criteria for determining whether a set in $E^{-}$is structure closed. In section 2 we consider the property of first countability for $\max V$.

Finally, in section 3 , we state and prove the main theorems. We show that if $V$ is separable, then $\max V$ is compact if and only if 0 does not belong to $Z$. We also show, for $V$ separable, that the properties of first countability, second countability, and local compactness are equivalent for $\max V$. 
This paper is a part of the author's doctoral dissertation submitted to Stanford University. The author would like to express. his appreciation to Professor Edward Effros for suggesting and discussing the problems investigated in this paper and to Professor Karel deLeeuw for his helpful suggestions in its preparation.

o. Conventions. The notation and definitions are those used in $[4,5,6]$. We shall use results freely from these papers. Throughout, we assume that vector spaces have nonzero elements. In normed spaces, the subscript $\lambda$ on a subset indicates the subset intersected with the closed ball of radius $\lambda$.

For any set $A \subseteq P_{1}(V), \bar{A}$ will denote the weak* closure of $A$. and $A^{+}$will denote $A-\{0\}$.

For any net, Greek subscripts, e.g., $\alpha, \beta$, denote arbitrary index sets while Latin subscripts, e.g., $i, j, n$, denote the natural numbers. as an index set, i.e., $\left\{x_{n}\right\}$ is a sequence.

For the entire paper, $V$ will always denote a separable simplex: space.

Throughout, propositions are stated in terms of the hull-kernel topology of $\max V$ and proven for the structure topology of $E$. Hopefully, this will cause no confusion.

1. The structure topology and several maps. In this section, we prove some preliminary results concerning the structure topology.

As $V$ is separable, $V_{1}^{*}$ is weak* metrizable and, thus, so is $P_{1}(V)$. As $P_{1}(V)$ is a simplex, Choquet's Theorem asserts that for each $q \in P_{1}(V)$ there is a unique maximal probability measure $\pi_{q}$ which represents $q$ and for which

$$
\pi_{q}\left(P_{1}(V)-E P_{1}(V)\right)=0 .
$$

$[10$, p. 70$]$. We shall always denote this measure by $\pi_{q}$.

Let $S[q]$ be the smallest weak* closed face of $P_{1}(V)$ containing0 and $q$, for $q \in P_{1}(V)$. As $S[q]$ is compact, metrizable, and convex, the Choquet Theorem applies equally as well to it. Hence, there is a maximal probability measure $\mu$ which represents $q$ and for which

$$
\mu(S[q] \text { - extreme points of } S[q])=0 \text {. }
$$

As $S[q]$ is a face, its extreme points are extreme in $P_{1}(V)$ and so

$$
\mu\left(S[q]-E P_{1}(V)\right)=0 .
$$

Since $P_{1}(V)$ is a simplex, $\mu=\pi_{q}$. Hence

$$
\operatorname{supp} \pi_{q} \subseteq S[q] \text {. }
$$


For $q \in P_{1}(V)$, we let $\operatorname{supp}^{+} \pi_{q}=\left\{y \in P_{1}(V)^{+} \mid\right.$each neighborhood $N \cong P_{1}(V)$ of $y$ satisfies $\left.\pi_{q}(N)>0\right\}$. Then, supp $\pi_{q}=\operatorname{supp} \pi_{q}-\{0\}$, consistent with our convention. The first proposition gives some relations between the structure and weak* topologies. Recall that for $A \subseteq P_{1}(V)$, the weak ${ }^{*}$ closure of $A$ is denoted by $\bar{A}$.

Proposition 1.1. (A) Let $q \in P_{1}(V)^{+}$. Then $\operatorname{supp}^{+} \pi_{q}$ is the closure in $P_{1}(V)^{+}$of supp $^{+} \pi_{q} \cap E^{+}$. Further,

$$
E^{+} \cap S[q]=\text { structure closure }\left(\operatorname{supp}^{+} \pi_{q} \cap E^{+}\right) \text {. }
$$

(B) Let $D \subseteq P_{1}(V)$. Suppose for each $q \in D$ we have supp $\pi_{q} \subseteq D$. If $D$ is weak* closed, then the weak* closed convex hull of $D$ is a face of $P_{1}(V)$ and $D \cap E^{+}$is structurally closed.

(C) Let $D \subseteq E^{+}$. Then the following are equivalent:

(1) $D$ is structure-closed.

(2) For each nonzero $q \in \bar{D}, S[q] \cap E^{+} \subseteq D$.

(3) For each nonzero $q \in \bar{D}$, $\operatorname{supp}^{+} \pi_{q} \cap E^{-} \subseteq D$.

(4) (a) $D$ is weak* closed relative to $E^{+}$.

(b) For each $q \in \bar{D}-E P_{1}(V)$, $\operatorname{supp}^{+} \pi_{q} \cap E^{\leftarrow} \subseteq D$.

Proof. (A) Since $P_{1}(V)^{+}$is a locally compact metric space,

$$
\pi_{q}\left(P_{1}(V)^{+}-\operatorname{supp}^{+} \pi_{q}\right)=0 .
$$

Since $\pi_{q}$ is a maximal measure,

$$
\pi_{q}\left(P_{1}(V)^{+}-E^{+}\right)=0 .
$$

Suppose $y$ is not in the closure in $P_{1}(V)^{+}$of $\operatorname{supp}^{+} \pi_{q} \cap E^{+}$. Then there is a relatively open set $N \cong P_{1}(V)^{+}$about $y$ such that

$$
N \cap\left(\operatorname{supp}^{+} \pi_{q} \cap E^{+}\right)=\varnothing \text {. }
$$

But then $N$ is open in $P_{1}(V)$ and obviously

$$
\pi_{q}(N) \leqq \pi_{q}\left(P_{1}(V)^{+}-\left(\operatorname{supp}^{+} \pi_{q} \cap E^{+}\right)\right)=0 .
$$

Thus $y \notin \operatorname{supp}^{+} \pi_{q}$. The other inclusion is trivial.

For the second conclusion, from the discussion preceeding the proposition,

$$
\operatorname{supp}^{+} \pi_{q} \cap E^{+} \subseteq S[q] \cap E^{+} .
$$

As the latter is structurally closed,

$$
\text { structure closure }\left(\operatorname{supp}^{+} \pi_{q} \cap E^{+}\right) \subseteq S[q] \cap E^{+} \text {. }
$$

For the other inclusion, let $K$ be the closed face containing zero which satisfies 


$$
K \cap E^{+}=\text {structure closure }\left(\operatorname{supp}^{+} \pi_{q} \cap E^{+}\right),
$$

which exists by the definition of the structure topology. As $K$ is closed and contains zero, the first part implies that supp $\pi_{q} \subseteq K$. As $K$ is convex, $q \in K$. But then $S[q] \subseteq K$ and so $S[q] \cap E^{+} \subseteq K \cap E^{+}$.

(B) The first conclusion is [5, Th. 3.3] while the second follows easily from the definition of the structure topology and the Milman Theorem [10, p. 9].

(C) $(1) \Rightarrow(2)$. Let $q$ be a nonzero element of $\bar{D}$. Then there is a sequence $\left\{p_{n}\right\} \subseteq D$ such that $p_{n} \rightarrow q$. Then $\left\{p_{n}\right\}$ tends structurally to each element of $S[q] \cap E^{+}\left[6\right.$, Lemma 2.3] and so $S[q] \cap E^{+} \cong D$.

$(2) \Rightarrow(3)$. Trivial.

$(3) \Rightarrow(4)$. Let $\left\{q_{n}\right\} \subseteq D$ and $q \in E^{+}$satisfy $q_{n} \rightarrow q$. Then $q \in \bar{D}$ and so supp $\pi_{q} \cap E^{+}=\{q\} \subseteq D$. Therefore $D$ is weak* closed relative to $E^{+}$.

$(4) \Rightarrow(1)$. Let $F=\bar{D} \cup\{0\}$. If $q \in F-\{0\}$, then $\operatorname{supp}^{+} \pi_{q} \cap E^{+} \subseteq D$ and so $\operatorname{supp}^{+} \pi_{q} \subseteq \bar{D}$ by part (A). Hence $\operatorname{supp} \pi_{q} \subseteq F$ and so (B) implies that $F \cap E^{+}$is structurally closed. Since $D$ is weak* closed in $E^{+}, F \cap E^{+}=\bar{D} \cap E^{+}=D$.

We must now define certain maps. Let $\widetilde{\Phi}: Z \rightarrow$ structure closed subsets of $E^{+}$by

$$
\widetilde{\Phi}(q)=S[q] \cap E^{+}
$$

If $0 \in Z$, then

$$
\widetilde{\Phi}(0)=\varnothing \text {. }
$$

We let $\tilde{\psi}: E^{+} \rightarrow$ subsets of $Z$ by

$$
\widetilde{\psi}(p)=\{q \in Z \mid p \in \widetilde{\Phi}(q)\} .
$$

Hence, for each $\mathrm{p} \in E^{+}$,

$$
0 \notin \widetilde{\psi}(p) .
$$

We extend these maps to set functions by letting

$$
\Phi(A)=\bigcup_{q \in A} \widetilde{\Phi}(q)
$$

for every set $A \subseteq Z$, and, for any $B \subseteq E^{+}$,

$$
\psi(B)=\bigcup_{p \in B} \widetilde{\psi}(p)=\{q \in Z \mid \widetilde{\Phi}(q) \cap B \neq \varnothing\} .
$$

For each $q \in Z$, we shall write $\Phi(q)$ to mean $\Phi(\{q\})$. Similarly, for each $p \in E^{+}$, we write $\psi(p)$ to mean $\psi(\{p\})$. Then, obviously, for $q \in Z$, 


$$
\Phi(q)=\widetilde{\Phi}(q)
$$

and for $p \in E^{+}$,

$$
\psi(p)=\tilde{\psi}(p)
$$

We may, alternately, describe the maps $\Phi$ and $\psi$ in terms of the relation $R$ in $E^{+} \times Z$ defined by

$$
p R q \text { if and only if } p \in S[q] \cap E^{+} .
$$

We have, for any $A \subseteq Z$,

$$
\Phi(A)=\{p \mid p R q, \text { some } q \in A\}
$$

and for any $B \subseteq E^{+}$,

$$
\psi(B)=\{q \mid p R q \text {, some } p \in B\} .
$$

Elementary relations for these maps are contained in the next lemma.

LEMMA 1.2. (1) For $A \subseteq Z^{+}, \psi \Phi(A) \supseteqq A$.

(2) For $B \subseteq E^{+}, \Phi \psi(B) \supseteqq B$.

(3) Let $p \in E^{+}$and $q \in Z$. Then $q \in \psi(p), p \in \Phi(q)$, and $p R q$ are equivalent.

(4) For $B \subseteq E^{+}, E^{+} \cap \psi(B)=B$.

(5) Let $q \in Z$. Then $\Phi(q)=$ structure closure $\left(\operatorname{supp}^{+} \pi_{q} \cap E^{+}\right)$.

Proof. They are all obvious.

If $A$ and $B$ are any topological spaces, a map $\Gamma: A \rightarrow$ subsets of $B$ is called lower semi-continuous if whenever $U \subseteq B$ is open then

$$
\{x \in A \mid \Gamma(x) \cap U \neq \varnothing\}
$$

is open in $A$ [2, Th. 1, p. 115].

Proposition 1.3. $\Phi$ is lower semi-continuous when $E^{+}$is given the structure topology. In fact, if $U \subseteq E^{+}$, then $U$ is structurally open if and only if $\psi(U)$ is weak* open in $Z$.

Proof. Let $U \subseteq E^{+}$. Then

$$
\begin{aligned}
Z-\psi(U) & =\{q \in Z \mid \Phi(q) \cap U=\varnothing\} \\
& =\left\{q \in Z \mid \Phi(q) \subseteq E^{+}-U\right\}
\end{aligned}
$$

Let us first suppose that $U$ is structurally open. Then $E^{+}-U$ is structurally closed. Hence, there is a closed face $K$ containing zero so that $K \cap E^{+}=E^{+}-U$. Let $q \in K \cap Z$. Then $S[q] \subseteq K$. Thus $\Phi(q) \subseteq E^{+}-U$ and so $q \in Z-\psi(U)$. If $q \in Z-\psi(U)$, then 
supp $\pi_{q} \subseteq\{0\} \cup \Phi(q) \subseteq\{0\} \cup\left(K \cap E^{+}\right) \subseteq K$ and so $q \in K$. Therefore, $Z-\psi(U)=K \cap Z$ and so $Z-\psi(U)$ is weak* closed.

Second, let us suppose that $\psi(U)$ is open. Then $(Z-\psi(U)) \cap E^{+}=$ $E^{+}-U$ is weak* closed in $E^{+}$. In order to show that $E^{+}-U$ is structure closed, we let $q$ be any nonzero element of $\overline{E^{+}-U}$. Since $\overline{E^{+}-U} \subseteq \overline{Z-\psi(U)} \cap \bar{E}^{+}=Z-\psi(U)$, we have that $S[q] \cap E^{+}=$ $\Phi(q) \subseteq E^{+}-U$. Hence, $E^{+}-U$ is structurally closed [Proposition $1.1(\mathrm{C})]$.

The main reason we introduced the map $\Phi$ is the following.

Proposition 1.4. Suppose $\left\{p_{n}\right\} \subseteq E^{+}$and $q \in Z$. If $p_{n} \rightarrow q$, then

(1) $F=\left\{p_{n}\right\} \cup \Phi(q)$ is structurally closed. to $F$.

(2) Let $p_{n_{0}} \in F-\Phi(q)$. Then $\left\{p_{n_{0}}\right\}$ is structurally open relative

(3) $\left\{p_{n}\right\}$ converges structurally to each element of $\Phi(q)$.

Proof. The conclusions are all trivial if $q=p_{N}=p_{\Lambda^{\prime} \div 1}=\cdots$ so we may assume that $\left\{p_{n}\right\}$ is not eventually the constant sequence $\{q\}$. For (1), we let $D=\left\{p_{n}\right\} \cup S[q]$. We claim that for each $z \in D$ we have $\operatorname{supp} \pi_{z} \subseteq D$. Indeed, if $z=p_{n_{0}}, \operatorname{supp} \pi_{p_{n_{0}}}=\left\{p_{n_{0}}\right\} \cong D$. If $z \in S[q]$, then $\operatorname{supp} \pi_{z} \subseteq S[z] \subseteq S[q]$. Since $S[q]$ is closed and $\left\{p_{n}\right\} \cup\{q\}$ is closed, $\bar{D}=D$. Therefore, $D \cap E^{+}=\left\{p_{n}\right\} \cup\left(S[q] \cap E^{+}\right)=\left\{p_{n}\right\} \cup \Phi(q)=$ $F$ is structurally closed [Proposition 1.1 (B)]. To show (2), we let $I=\left\{i \mid p_{i} \neq p_{n_{0}}\right\} . \quad$ As $k$ runs over $I, p_{k} \rightarrow q . \quad$ By $\operatorname{part}(1),\left\{p_{k} \mid k \in I\right\} \cup \Phi(q)$ is structurally closed, i.e., $\left\{p_{n_{0}}\right\}$ is structurally open relative to $F$. Part (3) is contained in [6, Lemma 2.3].

CoRollaRY 1.5. Suppose $\left\{p_{n}\right\} \subseteq E^{+}$and $q \in Z$. If $p_{n} \rightarrow q$, then the set of structure convergence points of $\left\{p_{n}\right\}$, the set of structure cluster points of $\left\{p_{n}\right\}$, and $\Phi(q)$ are the same set.

Proof. We need only show that if $x \in E^{+}$is a structure cluster point of $\left\{p_{n}\right\}$ then $x \in \Phi(q)$. Let $F=\left\{p_{n}\right\} \cup \Phi(q)$. As $F$ is structureclosed, $x$ a cluster point implies $x \in F$. Part (2) of the above shows that such an $x$ is not in $F-\Phi(q)$. Hence, $x \in \Phi(q)$.

2. Preliminary results. We study in this section the property of first countability for $\max V$. We shall derive several equivalent properties. Given that $\max V$ is first countable, we can find structure open sets by the following.

Lemma 2.1. Let $x \in E^{+}$and $W$ be any $w^{*}$-open set containing 
$\psi(x)$. If $x$ has a countable structure base, then $E^{+} \cap W$ has structure interior and, further, $x$ is in the structure interior of $\left(E^{+} \cap W\right)$.

Proof. The proof will be by contradiction. Let $U_{1} \supset U_{2} \supset \ldots$ be a structure base at $x$. Suppose that there is an $x_{n} \in U_{n}-\left(E^{+} \cap W\right)$. Then $\left\{x_{n}\right\}$ converges structurally to $x$. Since $Z$ is compact metric, there is a subsequence $\left\{x_{n_{k}}\right\}$ of $\left\{x_{n}\right\}$ and a point $y \in Z$ such that $x_{n_{k}} \rightarrow y$. Therefore $\mathrm{y} \in \psi(x)$ and so $y \in W$. But

$$
x_{n_{k}} \in\left(U_{n_{k}}-\left(E^{+} \cap W\right)\right) \cap E^{+}
$$

and so $x_{n_{k}} \notin W$ for each $n_{k}$. This contradicts the assumption that $W$ is a $w^{*}$-neighborhood of $y$.

Lemma 2.2. Let $x \in E^{+}$. Let $\widetilde{U}_{\varepsilon}=E^{+} \cap\{z \mid \operatorname{dist}(z, \psi(x))<\varepsilon\}$ and let $U_{\varepsilon}$ be the structure interior of $\tilde{U}_{\varepsilon}$. Assume $\psi(x)$ is compact and that $x \in U_{\varepsilon}$ for each $\varepsilon>0$.

Then, if $U$ is a structure neighborhood of $x$, there exists an. $\varepsilon>0$ such that $x \in U_{\varepsilon} \subseteq \widetilde{U}_{\varepsilon} \subseteq U$. In particular, $\left\{U_{1 / n}\right\}$ form $a$ countable structure base at $x$.

Proof. Let $U$ be a structure neighborhood of $x$. First note that $\psi(U)$ is a neighborhood of $\psi(x)$ [Proposition 1.3]. As $\psi(x)$ is compact, there is an $\varepsilon>0$ such that

$$
\psi(x) \subseteq\{z \mid \operatorname{dist}(z, \psi(x))<\varepsilon\} \subseteq \psi(U) .
$$

Intersecting with $E^{+}$, we have $x \in \widetilde{U}_{s} \subseteq \psi(U) \cap E^{+}=U$.

LEMma 2.3. Let $x \in E^{+}$and suppose that $x$ has a countable structure base. Then $\psi(x)$ is compact.

Proof. Since $Z$ is compact, it suffices to show that $\psi(x)$ is closed. Let $\left\{q_{n}\right\} \subset \psi(x)$ and $q \in Z$ satisfy $q_{n} \rightarrow q$. Let $O_{1} \supset O_{2} \supset \cdots$ be a $w^{*}$-base at $q$. We may assume that $q_{n} \in O_{n}$. Let $G_{1} \supset G_{2} \supset \ldots$ be a structure base at $x$. As $\psi(x) \subset \psi\left(G_{j}\right)$ and $\psi\left(G_{j}\right)$ is open $j=1,2, \cdots, \psi\left(G_{n}\right) \cap O_{n}$ is a $w^{*}$-open neighborhood of $q_{n}, n=1,2, \cdots$. Hence, there is a $p_{n} \in E^{+} \cap \psi\left(G_{n}\right) \cap O_{n}$. Consequently, $p_{n} \in G_{n}$ and so$\left\{p_{n}\right\}$ converges structurally to $x$. As $p_{n} \in O_{n}, \cdot p_{n} \rightarrow q$. But then $x \in \Phi(q)$, i.e., $q \in \psi(x)$ and so $\psi(x)$ is indeed compact.

Putting these three lemmas together yields the following.

CoRollary 2.4. Let $x \in E^{+}$and suppose that $x$ has a countable structure base. Let $\widetilde{U}_{\varepsilon}=E^{+} \cap\{z \mid \operatorname{dist}(z, \psi(x))<\varepsilon\}$ and let $U_{s}$ be the 
structure interior of $\tilde{U}_{\varepsilon}$. Let $U$ be any structure neighborhood of $x$. Then there exists an $\varepsilon>0$ such that $x \in U_{\varepsilon} \subseteq \widetilde{U}_{\varepsilon} \subseteq U$. In particular, $\left\{U_{1 / n}\right\}$ form a countable structure base at $x$.

If $C$ and $D$ are topological spaces, a map $\Gamma: C \rightarrow$ subsets of $D$ has a closed graph (at $c$ ) if whenever $\left\{c_{\alpha}\right\} \subseteq C$ converges to $c \in C$, $\left\{d_{\alpha}\right\} \subseteq D$ converges to $d \in D$, and $d_{\alpha} \in \Gamma\left(c_{\alpha}\right)$, then $d \in \Gamma(c)$.

We can now state and prove the main theorem in this section.

THEOREM 2.5. The following statements are equivalent:

(1) $\Phi$ has a closed graph.

(2) (a) $\psi(x)$ is compact for each $x \in E^{+}$.

(b) Let $x \in E^{+}$and $\widetilde{U}(\varepsilon)=\{z \mid \operatorname{dist}(z, \psi(x))<\varepsilon\} \cap E^{+}$. Then $x$ is in the structure interior of $E^{+} \cap \widetilde{U}(\varepsilon)$.

(3) $\max V$ is first countable.

(4) $\max V$ is second countable.

Proof. We shall show $(1) \Rightarrow(2) \Rightarrow(3) \Rightarrow(1)$ and $(3) \Rightarrow(4)$.

$(1) \Rightarrow(2)$. Let $x \in E^{+}$and $\widetilde{U}(\varepsilon)=\{z \mid \operatorname{dist}(z, \psi(x))<\varepsilon\} \cap E^{+}$. We first show that $x$ is in the structure interior of $\widetilde{U}(\varepsilon)$. Indeed, suppose that there is a net $\left\{x_{\alpha}\right\} \subseteq E^{+}-\widetilde{U}(\varepsilon)$ such that $\left\{x_{\alpha}\right\}$ converges structurally to $x$. Since $Z$ is compact, there is a subnet $\left\{x_{\alpha_{\beta}}\right\}$ and a point $y \in Z$ such that $x_{\alpha_{\beta}} \rightarrow y$. Since $x_{\alpha_{\beta}}=\Phi\left(x_{\alpha_{\beta}}\right)$, the closed graph condition implies that $x \in \Phi(y)$, i.e., $y \in \psi(x)$. Thus, dist $\left(x_{\alpha_{\beta}}, \psi(x)\right) \rightarrow 0$ and so $x_{\alpha_{\beta}} \in\{z \mid \operatorname{dist}(z, \psi(x))<\varepsilon\}$ eventually. It follows that $x_{\alpha_{\beta}} \in \widetilde{U}(\varepsilon)$ eventually and so $x_{\alpha} \in \widetilde{U}(\varepsilon)$ frequently. This contradicts the choice of $\left\{x_{\alpha}\right\}$ and so the claim is proven. Next, we must demonstrate that $\psi(x)$ is compact. In fact, we must only show that $\psi(x)$ is closed since $Z$ is compact. Let $\left\{y_{\alpha}\right\}$ be a net in $\psi(x)$ and $y \in Z$ satisfy $y_{\alpha} \rightarrow y$. The closed graph condition then implies that $x \in \Phi(y)$, i.e., $y \in \psi(x)$.

$(2) \Rightarrow(3)$ is Lemma 2.2 .

$(3) \Rightarrow(1)$. Suppose $\left\{x_{\alpha}\right\} \subseteq E^{+}, x \in E^{+}, \quad\left\{y_{\alpha}\right\} \cong Z$ and $y \in Z$. Let $y_{\alpha} \rightarrow y,\left\{x_{\alpha}\right\}$ converges structurally to $x$, and $x_{\alpha} \in \Phi\left(y_{\alpha}\right)$. We must show that $x \in \Phi(y)$. Let $\widetilde{U}_{n}=E^{+} \cap\{z \mid \operatorname{dist}(z, \psi(x))<1 / n\}$ and $U_{n}$ be the structure interior of $\tilde{U}_{n}$. Then $\left\{U_{n}\right\}$ forms a structure base at $x$ [Corollary 2.4]. Let $\left\{O_{n}\right\}$ be a $w^{*}$-base at $y$. Since $\left\{x_{\alpha}\right\}$ converges structurally to $x$, there is an $\alpha_{n}^{1}$ such that for each $\alpha \geqq \alpha_{n}^{1}, x_{\alpha} \in U_{n}$. Similarly, there is an $\alpha_{n}^{11}$ such that for each $\alpha \geqq \alpha_{n}^{11}, y_{\alpha} \in O_{n}$. Choose $\alpha_{n}$ larger than $\alpha_{n}^{1}, \alpha_{n}^{11}$, and $\alpha_{n-1}$. Taking $y_{n}=y_{\alpha_{n}}, x_{n}=x_{\alpha_{n}}$, clearly $y_{n} \rightarrow y,\left\{x_{n}\right\}$ converges structurally to $x, x_{n} \in \Phi\left(y_{n}\right) \cap U_{n}$. Recall that we must prove that $x \in \Phi(y)$.

Let 


$$
\widetilde{U}(\varepsilon, n)=E^{+} \cap\left\{z \mid \operatorname{dist}\left(z, \psi\left(x_{n}\right)\right)<\varepsilon\right\} .
$$

Since $x_{n} \in U_{n}$ and $U_{n}$ is a structure neighborhood about $x_{n}$, there is an $\varepsilon(n)>0$ such that $x_{n} \in \widetilde{U}(\varepsilon(n), n) \subseteq U_{n}$ [Corollary 2.4]. Let $\delta(n)=$ $\min (\varepsilon(n), 1 / n)$. Then $x_{n} \in \widetilde{U}(\delta(n), n) \subseteq U_{n}$. Since $E^{+}$is dense in $Z$, we can find $z_{n} \in E^{+}$such that dist $\left(z_{n}, y_{n}\right)<\delta(n) \leqq 1 / n$. Since $y_{n} \rightarrow y$, we have $z_{n} \rightarrow y$. Because $y_{n} \in \psi\left(x_{n}\right)$, we have $z_{n} \in E^{+} \cap\left\{z \mid \operatorname{dist}\left(z, \psi\left(x_{n}\right)\right)<\right.$ $\delta(n)\}=\tilde{U}(\delta(n), n)$. As $\tilde{U}(\delta(n), n) \subseteq U_{n}$, we have $z_{n} \in U_{n}$. Hence $\left\{z_{n}\right\}$ structurally converges to $x$. Thus, $x \in \Phi(y)$ [Corollary 1.5].

$(3) \Rightarrow(4)$. Let $\mathscr{S}=\left\{S_{i}\right\}$ be a countable base for $Z$. Let $x \in E^{+}$ and $U$ be an arbitrary structure neighborhood of $x$. Then $\psi(U)$ is a $w^{*}$-open neighborhood of each $y \in \psi(x)$. For each $y \in \psi(x)$, choose $S_{y} \in \mathscr{S}$ such that $y \in S_{y} \subseteq \psi(U)$. The neighborhoods $\left\{S_{y} \mid y \in \psi(x)\right\}$ cover $\psi(x)$. As $\psi(x)$ is compact [Lemma 2.3], a finite number of these neighborhoods cover $\psi(x)$. Thus,

$$
\psi(x) \subseteq \bigcup_{i=1}^{N} S_{y_{i}} \leqq \psi(U)
$$

But Lemma 2.1 implies that $x$ is in the structure interior of

$$
\left(E^{+} \cap \bigcup_{i=1}^{N} S_{u_{i}}\right)
$$

Since $E^{+} \cap \bigcup_{i=1}^{N} S_{y_{i}} \subseteq E^{+} \cap \psi(U)=U$, sets of the form

\{structure interior of $E^{+} \cap A \mid A$ is a finite union of sets from $\mathscr{S}$ \} form a countable structure base.

The proofs of Lemma 2.1 and $(3) \Rightarrow(4)$ are adapted from [12, Lemma 1 and Lemma $3, \S 4]$

3. The main theorems. The preceeding section has thrown light on several of the topological properties which we wanted to discuss. We will now discuss the others.

Though usually compactness and sequential compactness are not comparable for nonfirst-countable spaces, we have the following result:

Proposition 3.1 Let $K \subseteq \max V$. Then the following are equivalent:

(1) $K$ is compact.

(2) $K$ is sequentially compact.

Proof. $\quad(1) \Rightarrow(2)$. Let $K \cong E^{+}$be structurally compact and let 
$\left\{x_{n}\right\}$ be a sequence in $K$. Since $Z$ is compact metric, there is a subsequence $\left\{x_{n_{k}}\right\}$ and a point $y \in Z$ such that $x_{n_{k}} \rightarrow y$. As $K$ is structure-compact, the net $\left\{x_{n_{k}}\right\} \subseteq K$ has a cluster point $z \in K$. But then $z \in \Phi(y)$ [Corollary 1.5] and so $\left\{x_{n_{k}}\right\}$ converges structurally to $z \in K$ [Proposition 1.4]. Thus, $K$ is sequentially compact.

$(2) \Rightarrow(1)$. Let $K \subseteq E^{+}$be structurally sequentially compact and let $\left\{x_{\alpha}\right\}$ be a net in $K$. Since $Z$ is compact, there is a subnet $\left\{x_{a_{\beta}}\right\}$ and a point $y \in Z$ such that $x_{\alpha_{\beta}} \rightarrow y$. As $y$ has a countable neighborhood base, we may find a sequence $\left\{x_{n}\right\} \subseteq E^{+}$such that $x_{n} \rightarrow y$ and $\left\{x_{n}\right\} \subseteq\left\{x_{\alpha_{\beta}}\right\}$. Then $\left\{x_{n}\right\}$ is a sequence in $K$ and so there is a subsequence $\left\{x_{n_{k}}\right\}$ and a point $z \in K$ such that $\left\{x_{n_{k}}\right\}$ converges structurally to $z$. Then $z \in \Phi(y)$ and so $\left\{x_{\alpha_{\beta}}\right\}$ tends structurally to $z$ [6. Lemma 2.3] and so $K$ is compact.

We can now completely characterize those separable simplex spaces for which $\max V$ is compact.

THEOREM 3.2. The following are equivalent:

(1) $\max V$ is compact.

(2) $\max V$ is sequentially compact.

(3) $0 \notin Z$.

Proof. $\quad(1) \Longrightarrow(2)$ is a special case of Proposition 3.1.

$(2) \Longrightarrow(3)$. Suppose $0 \in Z$. Then there is a sequence $\left\{p_{{ }}\right\} \subseteq E^{+}$ such that $p_{n} \rightarrow 0$. Since $\max V$ is sequentially compact, there is a subsequence $\left\{p_{n_{k}}\right\}$ and a point $p \in E^{+}$such that $\left\{p_{n_{k}}\right\}$ tends structurally to $p$. But then Corollary 1.5 implies that $p \in \Phi(0)$. However $\Phi(0)=$ $\varnothing$ and we have a contradiction.

$(3) \Rightarrow(1)$. Let $\left\{U_{\alpha}\right\}$ be a structure-open cover of $E^{\div}$. Then $\mathbf{U}_{\alpha} U_{\alpha}=E^{+}$and so $\psi\left(\mathbf{U}_{\alpha} U_{\alpha}\right)=\mathbf{U}_{\alpha} \psi\left(U_{\alpha}\right)=\psi\left(E^{+}\right)=Z^{+}$. Since $0 \notin Z$, $Z^{+}=Z$ and so $\left\{\psi\left(U_{\alpha}\right)\right\}$ is an open cover of the compact set $Z$. Hence, there exists a finite subcover, i.e., there are sets $U_{\alpha_{1}}, \cdots, U_{\alpha_{N}}$ such that $\bigcup_{i=1}^{N} \psi\left(U_{\alpha_{i}}\right)=Z$. Then, intersecting both sides with $E^{+}$ yields

$$
E^{+}=\bigcup_{i=1}^{v}\left(E^{+} \cap \psi\left(U_{\alpha_{i}}\right)\right)=\bigcup_{i=1}^{N} U_{\alpha_{i}}
$$

[Lemma 1.2(4)]. Hence, $E^{+}$is structurally compact.

REMARK. The proof of $(3) \Rightarrow(1)$ establishes the fact that for a set $K \cong \max V$, if $\psi(K)$ is compact, then $K$ is compact.

We now come to the major result of this paper. 
THEOREM 3.3. Let $V$ be a separable simplex space. Then the following are equivalent:

(1) $\Phi$ has a closed graph.

(2) $\max V$ is first countable.

(3) $\max V$ is second countable.

(4) $\max V$ is locally compact.

Proof. Using Theorem 2.5, we must only show that (4) is equivalent to the others. We show first that first countability at one point implies local compactness at that point. Let $p \in E^{+}$and suppose $p$ has a countable structure neighborhood base. Let $U$ be a structure open set containing $p$. We must find a structurecompact neighborhood $K$ of $p$ within $U$. Let $F=E^{+}-U$. It is structurally closed and, hence, there is a closed face $Q$ of $P_{1}(V)$, containing zero, such that $Q \cap E^{+}=F$. Since $p \notin F, \psi(p) \cap Q=\varnothing$.

At this point, we shall specify the metric which we are using for $P_{1}(V)$. If $\left\{\xi_{n}\right\}$ is dense in $V_{1}$, then we take

$$
\operatorname{dist}(x, y)=\sum_{n} 2^{-n}\left|x\left(\xi_{n}\right)-y\left(\xi_{n}\right)\right| .
$$

Since $Q$ and $\psi(p)$ are compact [Lemma. 2.3],

$$
\operatorname{dist}(Q, \psi(p))=\delta>0
$$

Let

$$
D=\left\{z \in Z \mid \operatorname{dist}(\psi(p), z)<\frac{\delta}{2}\right\}
$$

and

$$
T=\left\{z \in Z \mid \operatorname{dist}(Q, z) \geqq \frac{\delta}{2}\right\}
$$

Then, clearly, $T$ is compact, $T \cap Q=\varnothing$, and $D \subseteq T$.

We first claim that $E^{+} \cap T$ is a structure neighborhood of $p$ within $U$. Indeed, $p \in E^{+} \cap D \subseteq E^{+} \cap T$ and $E^{+} \cap D$ is a structure neighborhood of $p$ [Lemma 2.1]. Obviously, $E^{+} \cap T \subseteq U$.

We next claim that $E^{+} \cap T$ is structure-compact. Let $\left\{p_{\alpha}\right\}$ be a net in $E^{+} \cap T$. Since $T$ is weak* compact, there is a subnet $\left\{p_{\alpha_{\beta}}\right\}$ and a point $q \in T$ such that $p_{\alpha_{\beta}} \rightarrow q$. Then $\left\{p_{\alpha_{\beta}}\right\}$ tends structurally to each point of $\Phi(q)$ [6, Lemma 2.3]. Hence it is sufficient to show $\Phi(q) \cap\left(E^{+} \cap T\right) \neq \varnothing$. For $z \in P_{1}(V)$, let 


$$
f(z)=\operatorname{dist}(z, Q) \text {. }
$$

Obviously $f$ is weak* continuous, $f(0)=0$ and $f(q) \geqq \delta / 2$. We next claim that $f$ is convex. Indeed, let $x, y \in P_{1}(V)$ and $0 \leqq \lambda \leqq 1$. We must demonstrate that

$$
f(\lambda x+(1-\lambda) y) \leqq \lambda f(x)+(1-\lambda) f(y) .
$$

Choose $q_{x}$ and $q_{y}$ in $Q$ such that

$$
f(x)=\operatorname{dist}\left(x, q_{x}\right)
$$

and

$$
f(y)=\operatorname{dist}\left(y, q_{y}\right) \text {. }
$$

Then

$$
\begin{aligned}
f(\lambda x+(1-\lambda) y) & =\operatorname{dist}(\lambda x+(1-\lambda) y, Q) \\
& \leqq \operatorname{dist}\left(\lambda x+(1-\lambda) y, \lambda q_{x}+(1-\lambda) q_{y}\right)
\end{aligned}
$$

since $\lambda q_{x}+(1-\lambda) q_{y} \in Q$ by convexity

$$
\begin{aligned}
& =\sum_{n} 2^{-n}\left|\lambda x\left(\xi_{n}\right)+(1-\lambda) y\left(\xi_{n}\right)-\lambda q_{x}\left(\xi_{n}\right)-(1-\lambda) q_{y}\left(\xi_{n}\right)\right| \\
& \leqq \sum_{n} 2^{-n}\left(\lambda\left|x\left(\xi_{n}\right)-q_{x}\left(\xi_{n}\right)\right|+(1-\lambda)\left|y\left(\xi_{n}\right)-q_{y}\left(\xi_{n}\right)\right|\right) \\
& =\lambda \sum_{n} 2^{-n}\left|x\left(\xi_{n}\right)-q_{x}\left(\xi_{n}\right)\right|+(1-\lambda) \sum_{n} 2^{-n}\left|y\left(\xi_{n}\right)-q_{y}\left(\xi_{n}\right)\right| \\
& =\lambda \operatorname{dist}\left(x, q_{x}\right)+(1-\lambda) \operatorname{dist}\left(y, q_{y}\right) \\
& =\lambda f(x)+(1-\lambda) f(y) .
\end{aligned}
$$

Since $S[q]$ is convex and weak* compact, $f$ restricted to $S[q]$ achieves its maximum at an extreme point of $S[q]$ [1, Satz 2]. As $S[q]$ is a face and $q \in S[q]$, there is a $p \in E P_{1}(V) \cap S[q]$ such that $f(p) \geqq f(q) \geqq$ $\delta / 2$. Since $f(0)=0$, there is a $p \in E^{+} \cap S[q]=\Phi(q)$ with $f(p) \geqq \delta / 2$. But this means that there is a $p \in \Phi(q) \cap T$. Thus, $E^{+} \cap T$ is structurally compact.

Next, we shall show that if $\max V$ is locally compact at a point, then that point has a countable neighborhood base. Let $x \in E^{+}$and assume that $E^{+}$is structurally locally compact at $x$. Let $\widetilde{U}(\varepsilon)=$ $\{z \in Z \mid \operatorname{dist}(z, \psi(x))<\varepsilon\} \cap E^{+}$and $U(\varepsilon)$ be the structure-interior of $\widetilde{U}(\varepsilon)$. We claim that $x \in U(\varepsilon)$. Indeed, suppose not. Then there is a net $\left\{x_{\alpha}\right\} \subseteq E^{+}-\widetilde{U}(\varepsilon)$ such that $\left\{x_{\alpha}\right\}$ tends structurally to $x$. Since $Z$ is compact, there is a subnet $\left\{x_{\alpha_{\beta}}\right\}$ and a point $y \in Z$ such that $x_{\alpha_{\beta}} \rightarrow y$. Suppose $x \notin \Phi(y)$. Let $U=E^{+}-\Phi(y)$. It is a structure open set containing $x$. By local compactness, there is a structure-compact neighborhood $K$ of $x$ in $U$. Therefore, $x_{\alpha_{\beta}} \in$ structure-interior $(K)$ 
for all $\alpha_{\beta} \geqq \alpha_{\beta_{0}}$. Then $\left\{x_{\alpha_{\beta}} \mid \alpha_{\beta} \geqq \alpha_{\beta_{0}}\right\}$ still converges to $y$. Since $Z$ is metrizable, we may select a sequence $\left\{x_{n}\right\} \subseteq E^{+}$such that $x_{n} \rightarrow y$ and $x_{n} \in\left\{x_{\alpha_{\beta}} \mid \alpha_{\beta} \geqq \alpha_{\beta_{0}}\right\}$. Then $\left\{x_{n}\right\} \subseteq$ structure-interior $(K)$ is a sequence in $K$. As $K$ is sequentially compact [Proposition 3.1], there is a subsequence $\left\{x_{n_{k}}\right\}$ and a point $z \in K$ such that $\left\{x_{n_{k}}\right\}$ tends structurally to $z \in K$. As $x_{n_{k}} \rightarrow y$, we have $z \in \Phi(y)$ [Corollary 1.5]. Thus $z \in \Phi(y) \cap K$, which contradicts the choice of $K$. Hence, $x \in \Phi(y)$, i.e., $y \in \psi(x)$. As $x_{\alpha_{\beta}} \rightarrow y$, dist $\left(x_{\alpha_{\beta}}, \psi(x)\right) \rightarrow 0$. Therefore $x_{\alpha_{\beta}} \in \widetilde{U}(\varepsilon)$ eventually and so $x_{\alpha} \in \widetilde{U}(\varepsilon)$ frequently. This contradicts the choice of the net $\left\{x_{\alpha}\right\}$ and so we have shown that $x \in U(\varepsilon)$.

In order to conclude that $x$ has a countable structure neighborhood base, we need only show that $\psi(x)$ is compact [Lemma 2.2]. As $Z$ is compact, we need only show that $\psi(x)$ is closed. Let $y \in \overline{\psi(x)}$. Suppose $y \notin \psi(x)$. Let $U=E^{+}-\Phi(y)$. It is a structure neighborhood of $x$ and so by hypothesis there is a compact neighborhood $K$ of $x$ in $U$. Let $G=$ structure interior $(K)$. Then $\psi(G)$ is a weak* neighborhood of $\psi(x)$. Since $y \in \overline{\psi(G)}$, we may choose $\left\{x_{k}\right\} \subseteq E^{+} \cap \psi(G)$ such that $x_{k} \rightarrow y$. Since $\left\{x_{k}\right\} \subseteq G \leqq K$, and $K$ is sequentially structurally compact [Proposition 3.1], there is a subsequence $\left\{x_{k_{j}}\right\}$ and there is a point $z \in K$ such that $\left\{x_{k_{j}}\right\}$ tends structurally to $z$. As $x_{k_{j}} \rightarrow y$, we have $z \in \Phi(y)$ [Corollary 1.5]. Thus, $z \in \Phi(y) \cap K$, contradicting the choice of $K$. Hence $y \in \psi(x)$ and so $\psi(x)$ is compact.

REMARK. The proof of Theorem 3.3 established a stronger equivalence than that stated. Namely, we showed that first countability at a point is equivalent to local compactness at that point.

Specializing Theorem 3.3. to the case when cardinality $\left(Z-E^{+}\right)<\infty$, we get the following.

CoRollary 3.4 Let $V$ be a separable simplex space. Suppose cardinality $\left(Z-E^{+}\right)<\infty$. Then $\max V$ is locally compact and second countable. Further, suppose $\left\{p_{\alpha}\right\}$ is a net in $E^{+}$converging weak* to $q$ and $p \in E^{+}$. Then the following are equivalent:

(1) $p \in \Phi(q)$.

(2) $\left\{p_{\alpha}\right\}$ converges structurally to $p$.

Proof. We first note that $\psi(p)$, for each $p \in E^{+}$, is a finite set and so is trivially compact. Fix $p \in E^{+}$and let $U_{\varepsilon}$ be the structureinterior of $\left\{z \in E^{+} \mid \operatorname{dist}(z, \psi(p))<\varepsilon\right\}$. If we show that $p \in U_{\varepsilon}$, then Theorem 2.5 allows us to conclude the first statement. Let

$$
A=\bigcup\left\{\Phi(q) \mid q \in Z-E^{+}, p \notin \Phi(q)\right\}
$$


and

$$
F=\left\{z \in E^{+} \mid \operatorname{dist}(z, \psi(p)) \geqq \varepsilon\right\} \cup A \text {. }
$$

Let $x$ be a nonzero element of $\bar{F}$. Suppose

$$
x \in \overline{\left\{z \in E^{+} \mid \operatorname{dist}(z, \psi(p)) \geqq \varepsilon\right\}} \text {. }
$$

Then $\operatorname{dist}(x, \psi(p)) \geqq \varepsilon$. If $x \in E^{+}$, then $\{x\}=\Phi(x) \subseteq F$. If $x \in Z-E^{+}$, then, since, $x \notin \psi(p)$, we have $\Phi(x) \subseteq A$. On the other hand, suppose $x \in \bar{A}$. As each $\Phi(q)$ is structurally-closed and cardinality $\left(Z-E^{+}\right)<\infty$, we have that $A$ is structurally closed. Thus, $\Phi(x) \subseteq A$ [Proposition 1.1 (C)]. Hence, we see that $F$ is structurally closed [Proposition 1.1 (C)] and so $E^{+}-F \cong U_{\varepsilon}$. As $p \notin F$, we conclude that $p \in U_{\varepsilon}$.

To show the second statement, we merely note that $(1) \Rightarrow(2)$ is [6, Lemma 2.3] and that $(2) \Rightarrow(1)$ is implied by $\Phi$ having a closed graph.

Specializing Theorem 3.3 to the case of $M$-spaces, we have the following.

THEOREm 3.5. Let $V$ be a separable $M$-space. Then the following are equivalent:

(1) Suppose $\left\{p_{n}\right\} \subseteq E^{+}$and $\left\{\lambda_{n} p_{n}\right\} \subseteq Z$. If $\left\{p_{n}\right\}$ converges and if $\lambda_{n} p_{n} \rightarrow 0$, then $p_{n} \rightarrow 0$.

(2) $\Phi$ has a closed graph.

(3) $\max V$ is first countable.

(4) $\max V$ is second countable.

(5) $\max V$ is locally compact.

(6) $\max V$ is metrizable.

Proof. Since $\max V$ for a separable $M$-space is normal [5, Th. 3.8], the equivalence of (2) through (6) follows from Theorem 3.3 and Urysohn's metrization theorem.

$(2) \Rightarrow(1)$. Since $\Phi(0)=\varnothing$, (1) is merely the statement that $\Phi$ has a closed graph at 0 , and so the implication is clear. In more detail, suppose $p_{n} \rightarrow y$ and $y \neq 0$. Let $z \in \Phi(y)$. Then $\left\{p_{n}\right\}$ tends structurally to $z$ [Proposition 1.4]. The closed graph condition implies that $z \in \Phi(0)=\varnothing$, which is a contradiction.

$(1) \Rightarrow(5)$. The map $\Phi: Z^{+} \rightarrow E^{+}$by

$$
\lambda p \rightarrow p
$$

is the factor map of $Z^{+}$, with the weak ${ }^{*}$ topology, onto $E^{+}$, with the 
structure topology [5, Formula 3.2 and .Th. 3.6]. Let $K$ be any compact set in $Z^{+}$. If $\Phi^{-1} \Phi(K)$ is compact for all such $K$, then $E^{+}$ is locally compact [3, Proposition 9, Ch. I, §10]. To show that $\Phi^{-1} \Phi(K)$ is compact, we note that

$$
\Phi^{-1} \Phi(K)=\{\lambda x \in Z \mid 0<\lambda \leqq 1, x \in K\} .
$$

As $Z$ is compact, it suffices to show that $\Phi^{-1} \Phi(K)$ is closed in $Z$. To show this, let $\left\{q_{n}\right\} \subseteq \Phi^{-1} \Phi(K)$ and $q \in Z$ satisfy $q_{n} \rightarrow q$. By definition, $q_{n}=\lambda_{n} x_{n}$ for some $x_{n} \in K$ and $0<\lambda_{n} \leqq 1$. Since $K$ and $[0,1]$ are compact metric, there is a subsequence $\left\{q_{n_{j}}\right\}$, an $x \in K$, and a $\lambda \in[0,1]$ such that $x_{n_{j}} \rightarrow x$ and $\lambda_{n_{j}} \rightarrow \lambda$. Hence, $q=\lambda x$. Since $x \in K$, if we show that $\lambda \neq 0$ then we have that $q \in \Phi^{-1} \Phi(K)$. However, if $\lambda=0$, then $q=0$ and so $q_{n_{j}} \rightarrow 0$. By (1), $x_{n_{j}} \rightarrow 0$. Since $K \cong Z^{+}$, this is impossible.

The results $(3) \Leftrightarrow(4) \Leftrightarrow(6) \Longrightarrow(5)$ are contained (tacitly, if not explicitly) in [5, Corollary $3.9 ; 12$, Th. 3].

We may now answer a question posed in [5]. Poulsen [11] has constructed a metrizable compact simplex $K$ for which $E^{+}$is dense in $K$, i.e., $Z=K$. Taking $V$ to be the affine functions on $K$ vanishing at 0 , we have a separable simplex space. Then $\max V$ cannot be locally compact. In fact, if $p \in E^{+}$, then $\lambda p \in Z$ for $0<\lambda \leqq 1$ and so $0 \in$ closure $(\psi(p))$. Hence, $\psi(p)$ is not compact and so $\max V$ is not locally compact at $M_{p}$ [Th. 3.3 and Th. 2.5]. More careful analysis shows that $\max V$ cannot contain even one compact set with interior.

It was conjectured in [5] that local compactness, first countability, isecond countability, and standard Borel structure were equivalent for separable simplex spaces. We have shown that the first three are indeed equivalent. As for the latter, we say that a Borel space has a standard Borel structure whenever it is Borel isomorphic to the Borel space associated with a Borel subset of a complete metric space [9, p. 138]. Since the extreme points of a metrizable compact convex set in a topological vector space form a $G_{i}$-set [10, Proposition 1.3], $E P_{1}(V)$ may be metrized by a complete separable metric [8, $\S 29, \mathrm{VI}]$. As $E^{+}$is a Borel subset of $E P_{1}(V), E^{+}$is standard. The map $M: p_{M} \rightarrow M$ is one-to-one, onto and continuous. Clearly, if $M^{-1}$ is a Borel function then $\max V$ is standard.

Proposition 3.6. If $\max V$ has a countable base, then $\max V$ is standard. Further, there is a separable $M$-space $V$ for which $\max V$ is standard but $\max V$ is not locally compact.

Proof. The first statement follows immediately from [9, Th. 3.2]. As for the second, let 


$$
V=\left\{f \in C[0,1] \mid f\left(\frac{1}{n}\right)=\frac{1}{n} f(1), n=2, \cdots\right\}
$$

$V$ is a separable $M$-space for which $\max V$ is not locally compact. [5, Th. 4.3]. By [5, Proposition 4.1],

$$
E^{-}=\left\{\hat{o}_{y} \mid 0<y \leqq 1 \text { and } y \neq \frac{1}{n} \text { for } n \geqq 2\right\}
$$

and

$$
Z=\left\{\delta_{y} \mid 0 \leqq y \leqq 1\right\}
$$

Therefore $Z-E^{-}$is a closed set. Hence, $E^{-}$is an open set in a compact metric space and so can be written as the countable union of compact sets, i.e., there are compact sets $K_{i} \subseteq Z$ such that

$$
E^{+}=\bigcup_{i} K_{i}
$$

For any set $A \subseteq E^{+}$, let

$$
M(A)=\bigcup\{M(p) \mid p \in A\} .
$$

Then $M(A)=\left(M^{-1}\right)^{-1}(A)$. Let $F$ be any closed set in $E^{-}$. Hence $M(F)=\bigcup_{i} M\left(K_{i} \cap F\right)$. Since $K_{i} \cap F$ is a compact set in $E^{+}, M\left(K_{i} \cap F\right)$ is closed [5, Corollary 3.5]. Thus, $M(F)$ is a countable union of closed sets in $\max V$ and so is Borel. Therefore, $M$ maps Borel sets to Borel sets, i.e., $M^{-1}$ is a Borel map. From above, this implies that $\max V$ is standard.

\section{REFERENCES}

1. H. Bauer, Minimalstellen von Funktionen und Extremalpunkte, Archiv. der Mathematik 9 (1958), 389-393.

2. C. Berge, Espaces topologiques, Dunod, Paris, 1966.

3. N. Bourbaki, Topologie generale, Actualites Scientifiques et Industrielles, No. 1142, Paris, 1965.

4. E. Effros, Structure in simplexes, Acta. Math. 117 (1967), 103-121.

5. - - Structure in simplexes II, J. Func. Anal. 1 (1967), 379-391.

6. E. Effros, and A. Gleit, Structure in simplexes III, Trans. Amer. Math. Soc. 142 (1969), 355-379.

7. J. Kelley, General topology, van Nostrand, Princeton, 1955.

8. C. Kuratowski, Topologie I, Monagrafie Mat. 20, Warsaw, 1958.

9. G. W. Mackey, Borel structures in groups and their duals, Trans. Amer. Math. Soc. 85 (1957), 134-165.

10. R. Phelps, Lectures on Choquet's theorem, van Nostrand, Princeton, 1966.

11. E. Poulsen, A simplex with dense extreme points, Ann. Inst. Fourier (Grenoble), 11 (1961), 83-87. 
12. A. H. Stone, Metrizability of decomposition spaces, Proc. Amer. Math. Soc. 7 (1956), 690-700.

Received October, 1, 1969. Prepared under the auspices of NSF Grant GP-8766.

Carnegie-Mellon University

Pittsburgh, Pennsylvania 



\title{
PACIFIC JOURNAL OF MATHEMATICS
}

\author{
EDITORS
}

\author{
H. SAMELSON \\ Stanford University \\ Stanford, California 94305 \\ RichaRd PIERCE \\ University of Washington \\ Seattle, Washington 98105
}

J. DugundJI

Department of Mathematics

University of Southern California

Los Angeles, California 9.0007

RICHARD ARENS

University of California

Los Angeles, California 9.0024

\section{ASSOCIATE EDITORS}

E. F. BeCKenBACH
B. H. NeumanN

F. WoLE

K. YoSHIDA

\section{SUPPORTING INSTITUTIONS}

\author{
UNIVERSITY OF BRITISH COLUMBIA \\ CALIFORNIA INSTITUTE OF TECHNOLOGY \\ UNIVERSITY OF CALIFORNIA \\ MONTANA STATE UNIVERSITY \\ UNIVERSITY OF NEVADA \\ NEW MEXICO STATE UNIVERSITY \\ OREGON STATE UNIVERSITY \\ UNIVERSITY OF OREGON \\ OSAKA UNIVERSITY \\ UNIVERSITY OF SOUTHERN CALIFORNIA
}

\author{
STANFORD UNIVERSITY \\ UNIVERSITY OF TOKYO \\ UNIVERSITY OF UTAH \\ WASHINGTON STATE UNIVERSITY \\ UNIVERSITY OF WASHINGTON \\ AMERICAN MATHEMATICAL SOCIETY \\ CHEVRON RESEARCH CORPORATION \\ TRW SYSTEMS \\ NAVAL WEAPONS CENTER
}

The Supporting Institutions listed above contribute to the cost of publication of this Journal, but they are not owners or publishers and have no responsibility for its content or policies.

Mathematical papers intended for publication in the Pacific Journal of Mathematics should be in typed form or offset-reproduced, (not dittoed), double spaced with large margins. Underline Greek letters in red, German in green, and script in blue. The first paragraph or two must be capable of being used separately as a synopsis of the entire paper. The editorial "we" must not be used in the synopsis, and items of the bibliography should not be cited there unless absolutely necessary, in which case they must be identified by author and Journal, rather than by item number. Manuscripts, in duplicate if possible, may be sent to any one of the four editors. Please classify according to the scheme of Math. Rev. Index to Vol. 39. All other communications to the editors should be addressed to the managing editor, Richard Arens, University of California, Los Angeles, California, 90024.

50 reprints are provided free for each article; additional copies may be obtained at cost in multiples of 50 .

The Pacific Journal of Mathematics is published monthly. Effective with Volume 16 the price per volume (3 numbers) is $\$ 8.00$; single issues, $\$ 3.00$. Special price for current issues to individual faculty members of supporting institutions and to individual members of the American Mathematical Society: $\$ 4.00$ per volume; single issues $\$ 1.50$. Back numbers are available.

Subscriptions, orders for back numbers, and changes of address should be sent to Pacific Journal of Mathematics, 103 Highland Boulevard, Berkeley, California, 94708.

PUBLISHED BY PACIFIC JOURNAL OF MATHEMATICS, A NON-PROFIT CORPORATION

Printed at Kokusai Bunken Insatsusha (International Academic Printing Co., Ltd.), 7-17, Fujimi 2-chome, Chiyoda-ku, Tokyo, Japan. 


\section{Pacific Journal of Mathematics}

\section{Vol. 34, No. $2 \quad$ June, 1970}

Shair Ahmad, On the oscillation of solutions of a class of linear fourth order

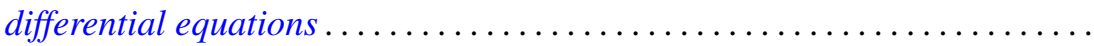

Leonard Asimow and Alan John Ellis, Facial decomposition of linearly

compact simplexes and separation of functions on cones ..............

Kirby Alan Baker and Albert Robert Stralka, Compact, distributive lattices of

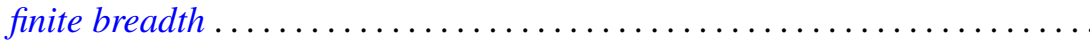

James W. Cannon, Sets which can be missed by side approximations to

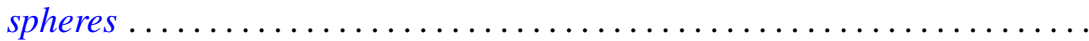

Prem Chandra, Absolute summability by Riesz means .................. 335

Francis T. Christoph, Free topological semigroups and embedding topological semigroups in topological groups....

Henry Bruce Cohen and Francis E. Sullivan, Projecting onto cycles in smooth, reflexive Banach spaces.................................

John Dauns, Power series semigroup rings .......................

Robert E. Dressler, A density which counts multiplicity ................

Kent Ralph Fuller, Primary rings and double centralizers ................

Gary Allen Gislason, On the existence question for a family of products.......

Alan Stuart Gleit, On the structure topology of simplex spaces .............

William R. Gordon and Marvin David Marcus, An analysis of equality in

certain matrix inequalities. $I \ldots \ldots \ldots \ldots \ldots \ldots \ldots \ldots$

Gerald William Johnson and David Lee Skoug, Operator-valued Feynman

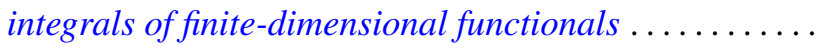

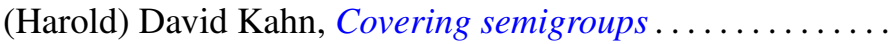

Keith Milo Kendig, Fibrations of analytic varieties

Norman Yeomans Luther, Weak denseness of nonatomic measures on perfect,

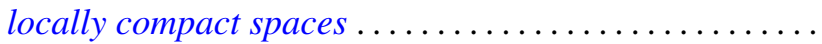

Guillermo Owen, The four-person constant-sum games; Discriminatory solutions on the main diagonal ...

Stephen Parrott, Unitary dilations for commuting contractions

Roy Martin Rakestraw, Extremal elements of the convex cone $A_{n}$ of

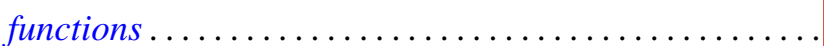

Peter Lewis Renz, Intersection representations of graphs by

William Henry Ruckle, Representation and series summability of complete

biorthogonal sequences.

F. Dennis Sentilles, The strict topology on bounded sets ...

Saharon Shelah, A note on Hanf numbers ...

Harold Simmons, The solution of a decision problem for several classes of rings. . .

Kenneth S. Williams, Finite transformation formulae involving the Legendre 